\title{
A Study on the Efficacy of Saline Nasal Irrigation in Chronic Rhinosinusitis
}

\author{
Rachana Ramachandran ${ }^{1}$, Santhi Thankappan Pillai ${ }^{2}$ \\ 1, 2 Department of ENT, Govt TD Medical College, Alappuzha, Kerala, India.
}

\section{ABSTRACT}

\section{BACKGROUND}

The objective of this study was to test as to whether daily saline nasal irrigation improved sinus symptoms in adult subjects with chronic rhino sinusitis, and also study the safety of saline irrigation, incidence of any complication and improvement in quality of life in these patients.

\section{METHODS}

This was a prospective observational study conducted between two groups of patients at Government TD Medical College, Alappuzha. Patients who satisfied the criteria for diagnosing chronic rhino sinusitis were included in the study. The first group was given saline nasal irrigation with oral drug therapy while the second group was put on oral drugs alone. Each subject was given a pretested, structured questionnaire. Along with socio-demographic information, the questionnaire also contained queries of quality of life measure, compliance of nasal irrigation and adverse effects following its use.

\section{RESULTS}

The pre-treatment scores and post treatment scores were analysed and the results were statistically significant with P values of $<0.001$ in all. $77 \%$ of the patients in the saline irrigation group were strictly using saline nasal irrigation which meant a satisfactory compliance and the improvement in post treatment scores was statistically highly significant with a P value of $<0.001$. Also there was a statistically significant difference between the mean pre-test and post test scores of the two groups with $\mathrm{P}$ value of $<0.001$ and $t$ value of 51.942 using the paired $t$ test.

\section{CONCLUSIONS}

Chronic rhinosinusitis has a major negative impact on the healthcare and economy of not only the patients but also of the society. Saline nasal irrigation is an effective yet easy method of therapy in alleviating the symptoms of chronic rhinosinusitis and improving the quality of life in these chronic sufferers.

\section{KEY WORDS}

Chronic Rhinosinusitis, Saline Nasal Irrigation, Quality of Life, Economic Burden
Corresponding Author:

Dr. Santhi T,

Associate Professor (ENT),

Department of ENT,

Govt TD Medical College,

Alappuzha, Kerala, India.

E-mail:sttpillai@gmail.com

DOI: $10.14260 / j e m d s / 2021 / 463$

How to Cite This Article:

Ramachandran R, Pillai ST. A study on the efficacy of saline nasal irrigation in chronic rhinosinusitis. J Evolution Med Dent Sci 2021;10(30):2266-2270, DOI: $10.14260 / \mathrm{jemds} / 2021 / 463$

Submission 03-02-2021,

Peer Review 20-05-2021,

Acceptance 27-05-2021,

Published 26-07-2021.

Copyright ( $(2021$ Rachana Ramachandran et al. This is an open access article distributed under Creative Commons Attribution License [Attribution 4.0 International (CC BY 4.0)] 


\section{BACKGROUND}

Sinusitis refers to a group of disorders characterised by the inflammation of the mucosa of the PNS.1,2 The inflammation almost always involves the nose along with the sinuses and hence the preferred term is' Rhinosinusitis'. Rhinosinusitis Task Force (RSTF) has mentioned a group of symptoms for the clinical diagnosis of rhinosinusitis, the criteria being either 2 major symptoms / 1 major symptom+ 2 minor symptoms. 2,3 The major and minor symptoms are classified in Table $1 .{ }^{3}$

\begin{tabular}{|cc|}
\hline Major Symptoms & Minor Symptoms \\
Facial pain / pressure / fullness & Fever (other than acute sinusitis) \\
Nasal obstruction / blockage & Halitosis \\
Nasal or postnasal discharge or purulence & Fatigue \\
as by history or examination & Dental pain \\
Hyposmia / anosmia & Cough \\
Purulence on nasal examination & Ear pain / pressue / fullness \\
Fever(acute only) & Table 1. Rhinosinusitis Symptoms / Signs \\
\hline
\end{tabular}

Chronic rhinosinusitis (CRS) was defined by the original rhinosinusitis task force (RSTF) in 1997 by the inclusion of 2 or more major factors or 1 major and 2 minor factors for more than 12 weeks. In 2007 based on the guidelines issued by the American Academy of Otolaryngology (AAO) the definition of rhinosinusitis was updated requiring either radiographic or endoscopic evidence of inflammation in addition to the presence of 2 or more RSTF major criteria of symptoms. ${ }^{4,5}$ CRS which is defined as mucosal inflammation of the nose and paranasal sinuses and may be divided into two broad clinical categories such as CRS with and without nasal polyposis. ${ }^{6}$ In India about $15 \%$ of the population is affected by rhinosinusitis. ${ }^{7}$ Glicklich et al. ${ }^{8}$ have shown that rhinosinusitis has a significant impact on the Quality of Life (QOL) even in comparison to chronic debilitating diseases such as diabetes and congestive heart failure. The RSDI (Rhinosinusitis disability index) is a validate instrument that measures the physical, functional and emotional impact of rhinosinusitis on a person's QOL. ${ }^{9}$ It measures 30 parameters with scores ranging from 0 - 120 .

There is inflammation of the nasal mucosa in chronic rhinosinusitis and the associated serous, mucus and mucopurulent secretions from the nasal mucosa become an ideal growth medium for the various pathogens like virus, bacteria, fungus etc. Therefore removal of these secretions would lead to a significant relief on the natural course of CRS. ${ }^{10}$ The ability of the paranasal sinuses to clear the mucous secretions depends on the patency of the sinus ostia, the mucociliary action and the consistency of the mucous. Once the sinus mucosa gets irritated by the etiological factors in CRS, inflammatory oedema, sinus outflow block, mucociliary dysfunction with proliferation of the pathogens ensue relentlessly. ${ }^{4}$

Medical management of CRS includes short and long-term antibiotic therapy, topical and systemic steroids, topical and oral decongestants, oral antihistamines, mast cell stabilisers, anti-leukotriene agents, mucolytics, topical antibiotics, topical and systemic antimycotics, proton pump inhibitors, bacterial lysates, immunotherapy, phytotherapy targeted bio therapeutic agents like anti - IgE and anti-cytokine antibodies and avoidence of environmental factors. ${ }^{11,12,13}$

Saline nasal irrigation (SNI) can be used as an adjunctive measure in CRS.10 Originally described in the ayurvedic medical treatment as 'jala neti' this method was later taken up by the Western medicine in the late 19th century.

The Lancet published in 1902 the indications and usage of SNI. Wingrave described nasal douching methods in $1902 . .^{14}$ Proetz described isotonic saline irrigation of the nose and sinuses in his book "The displacement method of sinus diagnosis and treatment". 15 Clinical trials have supported its use not only in CRS, but also as an adjunctive therapy for upper respiratory infections and allergic rhinitis. ${ }^{16}$ Though it is prescribed in everyday clinical practice, NI is mentioned very briefly in the guidelines for treatment of upper tract infections. ${ }^{12}$ The first study to assess the use of SNI as an adjunctive therapy for upper respiratory infections by the family physicians in Wisconsin was conducted among the 286 physicians and $90 \%$ recommended SNI in CRS. ${ }^{16}$

SNI reduces nasal dryness and facilitates clearing of thick mucus and crusts from the nasal cavity. However, there is an increasing perception that saline has a contributory role in the resolution of inflammation as well. Many theories exist for the potential beneficial physiological effects of topical saline. Improvement in mucus clearance, enhanced ciliary beat activity, removal of antigen, biofilm or inflammatory mediators and a protective role on sino - nasal mucosa have all been proposed.10 Addition of ions like magnesium, zinc, potassium and bicarbonate to saline have shown to have positive effects like promoting cell repair, reducing apoptosis and mucus viscosity etc. ${ }^{12}$

\section{Objectives}

1. To study the effectiveness of the use of saline nasal irrigation (SNI) in routine primary care for chronic or recurrent sinus symptoms.

2. Also to study the safety of SNI, incidence of any complication and improvement in the quality of life in these patients.

\section{METHODS}

This is a prospective observational study which was conducted on the patients attending the ENT outpatient department, at T D Medical College, Alappuzha from January 2015 to December 2015. After getting clearance from the Institutional Research and Ethical Committee, 400 patients diagnosed with CRS were enrolled as per the inclusion and exclusion criteria. Signed written informed consent was obtained from all the 400 patients included in the study. Detailed evaluation was done which included ENT examination and systemic examination. Diagnostic Nasal Endoscopy was done for all patients. At the initial visit a baseline RSDI score was calculated for all and it was recorded (Table 2). Both groups were asked to fill a questionnaire and grade their symptoms. This was called as the pre-treatment score. The 200 patients under Group I received SNI daily in addition to oral medication for symptomatic relief with antibiotics, decongestants and antihistamines, while the remaining 200 belonging to Group II were given oral antibiotics, decongestants and antihistamines alone. All patients were asked to review after 4 weeks. Those in Group I were given a detailed class on the method of SNI. Each patient was given a $20 \mathrm{cc}$ plastic syringe with a cannula, mesuring 
spoon, non-iodised salt and a container. The patients were asked to prepare the saline solution in the following manner.

- Put 1 teaspoon of salt into the container.

- Add $500 \mathrm{ml}$ of lukewarm water.

- Add a pinch of sodium bicarbonate to make it physiological.

- Mix the contents.

Instructions were also given for the method of saline irrigation as detailed below

- Lean over the sink. Turn your head to one side.

- Insert the cannula tip into the upper most nostril and breathe through your mouth.

- Push gently the piston of the syringe so that the solution flows into the upper nostril

- In a few moments, the solution will begin to drain from the lower nostril.

- $\quad$ Continue until the syringe is empty, then exhale gently through both nostrils.

- $\quad$ Refill the syringe, turn your head to the opposite side, and repeat in the other nostril.

- Do this two times daily for 4 weeks.

The patients were told to clean the syringe and cannula with warm water and a mild detergent. The unused saline solution was to be kept in a sealed container at room temperature for the next 48 hours.

\section{Statistical Analysis}

The same questionnaire was filled at the end of 4 weeks of treatment and the total score was calculated. This was called the post treatment score. Qualitative variables were expressed in percentages or proportion and quantitative variables summarised in mean with standard deviation. Chi square test was used for testing the significance. Paired t test was used to find the significance of the post treatment score between the two groups.

\section{Inclusion Criteria}

All patients in the age group between 16 and 76 years with 2 major symptoms / one major +2 minor symptoms persisting for more than 12 consecutive weeks. The symptoms of CRS was less in patients below 16 years and compliance to saline nasal irrigation was less in patients below 16 years and above 76 years.

\section{Exclusion Criteria}

Patients presenting with the following conditions were excluded.

- Marked degree of DNS

- Extensive nasal polyposis

- Atrophic Rhinitis

- Severe facial trauma

- History of nasal surgery in the past

\section{Sample Size}

Sample size was calculated by the statistical formula $n=z^{2} p(1$ - p) / $d^{2}$ where $n$ is the sample size, $z$ is the value of the standard normal distribution of $1.96, \mathrm{p}$ is the expected proportion of the population (0.15) and $\mathrm{d}$ is the absolute precision of $1 / 20(0.05)$, according to the prevlence rate of rhinosinusitis by $15 \%$ in India. $^{7}$

\section{RESULTS}

There were 400 patients with a diagnosis of CRS and fitting within the inclusion criteria. During the course of the study, all were followed up for four weeks. 200 patients were given symptomatic treatment with oral antibiotics, decongestants and antihistamines, while the rest 200 received daily saline nasal irrigation (SNI) in addition to the oral medications. At the initial visit a baseline RSDI score was calculated for all and recorded. They were given respective treatment randomly and asked to review after 4 weeks. The main focus of the study was to find out whether SNI would improve the symptoms in CRS.

Age wise, the youngest patient was 18 years old and the oldest 60 . Most number of patients belonged to the $30-40$ years of age group. The mean age was 39.91 with a standard deviation of 11.685 (Table 2).

\begin{tabular}{|ccc|}
\hline Age Group & Frequency (Number) & Percentage \\
< 30 years & 95 & 23.75 \\
30 - 40 years & 136 & 34 \\
40 - 50 years & 51 & 12.75 \\
50 - 60 years & 118 & 29.5 \\
Total & 400 & 100 \\
\hline \multicolumn{2}{|c|}{ Table 2. Age Distribution of the Study Population } \\
\hline
\end{tabular}

Males constituted $47.2 \%$ and females constituted $52.8 \%$ of the study population. Any gender based difference in RSDI score after treatment was analysed but showed no statistical significance (chi-square - 0.973). The main clinical symptoms were tabulated (figure 1). The most frequent symptom was nasal block ( $45.8 \%$ ) followed by facial pain (23\%), hyposmia (12.8\%), nasal discharge (11.2\%), headache (6.8\%) and the least frequent symptom was anosmia (0.5\%).

Among the patients in the study group, there were 143 graduates (35.8\%), 141 postgraduates (35.2\%) and 116 (29 $\%)$ patients had only elementary schooling. The association between the educational status and the post treatment score in the two groups was analysed, but was not significant with a chi-square value of 0.011 , implying that the method of using nasal irrigation was simple and easily practicable to all patients irrespective of their educational status.

On analysing the pre-treatment and post treatment symptoms of patients with CRS of Group I and Group II, Group I patients who were treated with saline nasal irrigation in addition to oral medication showed much more relief in the symptoms of headache, facial pain and nasal discharge than those in Group II who were on oral medication alone, which was statistically significant with a P value of $<0.001$ in all. This implied that patients with these symptoms pertaining to CRS were very well benefitted by SNI (Table 3).

Also there was a statistically significant difference between the mean pre-test and post test scores of the two groups with a $\mathrm{P}$ value of $<0.001$ and with a $t$ value of 51.942 using the paired t test. $77 \%$ of the patients in group I were strictly using SNI, while $23 \%$ were occasional users which indicated a satisfactory compliance (Table 4). Side effects were few, mainly nasal irritation (17\%), pooling of saline (11\%), headache (3\%) and epistaxis ( $1 \%$ ), while $68 \%$ of the study 
population did not have any adverse effect on using nasal irrigation.

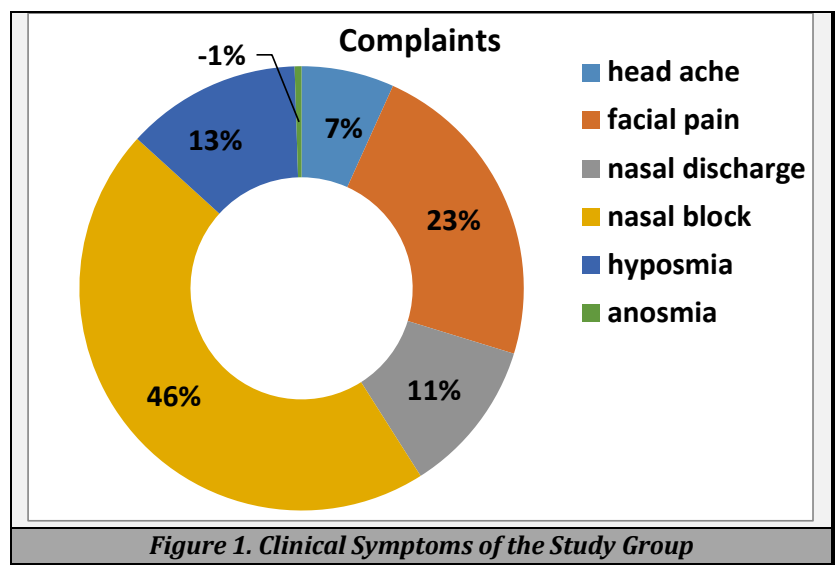

\begin{tabular}{|cccc|}
\hline $\begin{array}{c}\text { Efficacy of } \\
\text { Treatment by SNI }\end{array}$ & $\begin{array}{c}\text { Group I (SNI } \\
\text { +Oral } \\
\text { Medication) }\end{array}$ & $\begin{array}{c}\text { Group II(Oral } \\
\text { Medication } \\
\text { Alone) }\end{array}$ & $\begin{array}{c}\text { Marginal Row } \\
\text { Total }\end{array}$ \\
Improvement & 128 & 59 & 187 \\
No improvement & 72 & 141 & 213 \\
Marginal column total & 200 & 200 & 400 \\
\hline \multicolumn{4}{|c|}{ Table 3 Efficacy of Saline Nasal Irrigation } \\
Chi - square test: 47.812 P - value is $<0.00001$. Significant at P $<0.05$ \\
\hline
\end{tabular}

\begin{tabular}{|ccc|}
\hline Usage of SNI & Frequency & Percentage $\%$ \\
Occasional use & 46 & 23 \\
Strict use & 154 & 77 \\
Total & $\mathbf{2 0 0}$ & $\mathbf{1 0 0}$ \\
\hline Table 4. Use of Saline Nasal Irrigation in the Study Population \\
\hline
\end{tabular}

\section{DISCUSSION}

Saline irrigation has gained popularity in relieving the symptoms of chronic sinusitis and there have been several randomized controlled trials showing objective and subjective efficacy of saline irrigation in sinonasal diseases. ${ }^{12}$ The main purpose of the study was to find out if SNI improved the symptoms of CRS, increased QOL thereby reducing the overall morbidity of the disease. Moreover saline being cheap and physiological, is highly safe and has very minimal side effects; thus it could be practised in our setting and thereby decreases the recurrence and duration of medications for CRS. $0.9 \%$ to 3 $\%$ saline solutions have been used most often. Though optimal $\mathrm{pH}$ and temperature are not known, ${ }^{14} 4.5$ to $7 \mathrm{pH}$ is recommended. ${ }^{12}$ Harvey et al. was the first to analyze the clinical relevance of the therapeutic use of nasal saline irrigation in chronic rhinosinusitis which was published in a Cochrane review in 2007.12 This included 8 randomized controlled trials. These studies could not be standardised as there were differences in the selection of patients, methods used for nasal irrigation and in evaluating the outcome measures. Efficacy of nasal irrigation with saline solution was compared with a placebo, with no treatment or as an adjunct with other treatment. Comparison between hypertonic saline solution and isotonic solution was also done. Patients had similar improvement with both hypertonic and isotonic saline solutions, but radiological scores seemed better in patients after hypertonic saline therapy.

On comparing nasal irrigation with hypertonic and isotonic saline irrigation in 40 patients by using $200 \mathrm{ml}$ of solution twice a day, Bachman et al. noted similar clnical improvement in both the groups but Hauptman and Ryan who studied on 80 patients found that nasal airway improvement was better with isotonic saline nasal irrigation. ${ }^{12}$ The mode of saline administration is also important where large volume low pressure isotonic saline irrigation was found to be more effective than saline nasal spray in reducing the use of medication and thereby improving the quality of life.17 Similarly in the prospective RCT comparing nasal spray and nasal irrigation by KIM HM et al. ${ }^{17}$ symptom and severity and disease specific quality of life were assessed with the sinonasal outcome test (SNOT - 20) which is a 20 item survey that measures physical problems, emotional consequences and functional limitations of sinusitis. ${ }^{18}$ The conclusion was that the saline irrigation group had lower SNOT - 20 scores than the nasal spray group.

However, addition of isotonic saline irrigation after 3 months in the postoperative cases did not reduce the crusting, oedema, adhesions, and polyps in the study of 23 patients conducted by Freeman et al. ${ }^{2}$ Khianey et al. reported that nasal irrigation could reduce the use of medicines and therefore minimizes resistance to antibiotics. ${ }^{19}$ In the study group of 400 patients, 189 were males and 211 females. The different age and sex distributions were studied but it showed no significant association with the outcome. The educational status of the population also did not affect the outcome thereby favouring easy use of SNI in all. $77 \%$ of the patients in the second group were strictly using SNI which meant a satisfactory compliance and the improvement in post treatment scores in them was statistically highly significant with a $P$ value of $<0.001$.

SNI, among all the complaints could maximally improve the symptoms of nasal block, facial pain and head ache. The relationship between various symptoms and post treatment score was analysed and the results were statistically significant in those with headache, facial pain, nasal discharge with $P$ values of $<0.001$ in all implying that patients' symptoms pertaining to CRS were benefitted by SNI. There was a statistically significant difference between the mean pre-test and post test scores of the two groups with a $\mathrm{P}$ value of $<0.001$ with a $t$ value of 51.942 using the paired $t$ test. The study was successful in this regard by getting a statistically significant difference between the two groups after treatment which was comparable to previous similar studies. The study also analysed the side effect profile of SNI which showed nasal irritation as the major adverse effect in $17.2 \%$ of the total population others being pooling of saliva, headache and epistaxis.

Among the 400 patients who took part in the study, all received some form of treatment and both were successful in improving the symptoms of CRS which was comparable to various studies done before and hence SNI is established as an adjuvant therapy in CRS. Other than CRI, SNI is also recommended as an adjunctive therapy in acute upper respiratory tract infections, allergic rhinitis, rhinitis of pregnancy and Wegener's granulomatosis. SNI is also advocated in certain other conditions like in elderly people with sinusitis, ${ }^{20}$ rhinitis medicamentosa, ${ }^{21}$ infants with nasolacrimal duct obstruction (in decontaminating the nose following industrial accidents, ${ }^{22}$ after nasal tumour removal ${ }^{23}$ and choanal atresia repair. ${ }^{24}$ Looking into the emerging trends in topical therapy, surfactants which reduce water surface 
tension may be used to dissolve biofilms. $1 \%$ baby shampoo in normal saline has been used in inhibiting the biofilm formation of pseudomonas species in vitro. Xylitol and sodium hypochlorite are the newer additives for nasal irrigation. Both are tolerated well and give good symptomatic relief, disease clearance as well as in endoscopic appearance. ${ }^{11}$

\section{CONCLUSIONS}

Rhinosinusitis is a worldwide health issue causing significant morbidity. Saline nasal irrigation is a very useful, yet inexpensive method with little complications to alleviate the symptoms related to CRS. The method of irrigation also has a high compliance. The technique of saline nasal irrigation should be standardised and should be considered in all patients with CRS along with the medical and surgical management for maximum benefit. The easily prepared solution of saline douche can also be used as a home remedy, thereby, avoiding prolonged hospital stay. This is one technique which can be used widely regardless of the age or comorbidities of the general population with CRS.

\section{Limitations}

All data were obtained from the patients and hence subjected to recall bias. The patients were followed up only for a period of 4 weeks. The long term efficacy SNI in CRS was not studied. Quality of life assessment (QOL) was done indirectly using symptom index and the impact of CRS on the subjects' productivity was not entirely measured, as no information was available on daily work performance. Effectiveness of SNI was not studied in the paediatric age group who are at a high risk of frequent upper respiratory tract infections.

Data sharing statement provided by the authors is available with the full text of this article at jemds.com.

Financial or other competing interests: None.

Disclosure forms provided by the authors are available with the full text of this article at jemds.com.

\section{REFERENCES}

[1] Rosenfeld RM, Andes D, Bhattacharyya N, et al. Clinical practice guidelines on adult sinusitis. Otolaryngol Head Neck Surg 2007;137(3 Suppl):375-7.

[2] Meltzer EO, Hamilos DL. Rhinosinusitis diagnosis and management for the clinician: a synopsis of recent consensus guidelines. Mayo Clin Proc 2011;86(5):427-43.

[3] Hadley JA, Schaefer SD. Clinical evaluation of rhinosinusitis: history and physical examination. Otolaryngol Head Neck Surg 1997;117(3 pt 2):S8-11.

[4] Lanza DC, Kennedy DW. Adult rhinosinusitis defined. Otolaryngol Head Neck Surg 1997;117(3 Pt 2):S1-7.

[5] Rosenfeld RM, Piccirillo JF, Chandrasekhar SS, et al. Clinical practice guideline (update): adult sinusitis. Otolaryngol Head Neck Surg 2015;152(2 Suppl):S1-39.
[6] Casale M, Moffa A, Cassano M, et al. Saline nasal irrigations for chronic rhinosinusitis: from everyday practice to evidence-based medicine. An update. Int J Immunopathol Pharmacol 2018;32:2058738418802676.

[7] Sood VP. Chronic Rhinosinusitis. ECAB clinical update: otorhinolaryngology. $1^{\text {st }}$ edn. Elsevier Health Sciences 2011:75-83

[8] Glicklich RE, Metson R. The health impact of chronic sinusitis in patients seeking otolaryngologic care. Otolaryngol Head Neck Surg 1995;113(1):104-9.

[9] Benninger MS, Senior BA. The development of the rhinosinusitis disability index. Arch Otolaryngol Head Neck Surg 1997;123(11):1175-9.

[10] Gupta V, Gupta M, Singh S. Nasal saline irrigation for chronic rhinosinusitis nasal saline irrigation for chronic rhinosinusitis 1. Clinical Rhinology 2010;3(3):145-7.

[11] Cain RB, Lal D. Update on the management of chronic rhinosinusitis. Infect Drug Resist 2013;6:1-14.

[12] Principi N, Esposito S. Nasal irrigation: an imprecisely defined medical procedure. Int J Environ Res Public Health 2017;14(5):516.

[13] Gurrola J, Borish L. Chronic rhinosinusitis: endotypes, biomarkers and treatment response. J Allergy Clin Immunol 2017;140(6):1499-1508.

[14] Wingrave W. The nature of discharges and douches. Lancet 1902;159(4107):1373-5.

[15] Proetz AW. The displacement method of sinus diagnosis and treatment: a practical guide to the use of radiopaques in the nasal sinuses, with 146 illustrations and a chart. St. Louis: Annals Publishing Co., 1931.

[16] Rabago D, Zgierska A. Saline nasal irrigation for upper respiratory conditions. Am Fam Physician 2009;80(10):1117-9.

[17] Pynnonen MA, Mukerji SS, Kim HM, et al. Nasal saline for chronic sinonasal symptoms: a randomized controlled trial. Arch Otolaryngol Head Neck Surg 2007;133(11):1115-20.

[18] Piccirillo JF, Merritt MG Jr, Richards ML. Psychometric and clinimetric validity of the 20-Item Sino-Nasal Outcome Test (SNOT-20). Otolaryngol Head Neck Surg 2002;126(1):41-7.

[19] Papsin B, McTavish A. Saline nasal irrigation: its role as an adjunct treatment. Can Fam Physician 2003;49:168-73.

[20] Knutson JW, Slavin RG. Sinusitis in the aged. Optimal management strategies. Drugs Aging 1995;7(4):310-6.

[21] Pietrusko RG, Anderer TJ. Oculoplastic update: drug information forum: rebound congestion from topical nasal decongestants. 1980;5:16.

[22] Berger ME, Jones OW, Ricks RC, et al. Decontaminating the nasal passages. Health Physics 2003;84(5 Suppl):S80-2.

[23] Pope TH Jr. Surgical approach to tumors of the nasal cavity. Laryngoscope 1978;88(11):1743-8.

[24] Holzmann D, Ruckstuhl M. Unilateral choanal atresia: surgical technique and long-term results. J Laryngol Otol 2002;116(8):601-4. 